OPEN ACCESS

Edited by:

Won Fen Wong,

University of Malaya, Malaysia

Reviewed by:

Piriyaporn Chongtrakool,

Mahidol University, Thailand

Hyeri Seok,

Korea University Medical Center,

South Korea

Retno Wahyuningsih,

Universitas Kristen Indonesia, Indonesia

*Correspondence: Youzhen Qin

qyouzhen@163.com

Specialty section:

This article was submitted to Microbial Immunology,

a section of the journal

Frontiers in Immunology

Received: 25 March 2021 Accepted: 04 June 2021

Published: 21 June 2021

Citation:

Ba $H$, Peng $H$, Cheng L, Lin $Y, L i X$, He X, Li S, Wang H and Qin Y (2021) Case Report: Talaromyces marneffei Infection in a Chinese Child With a Complex Heterozygous CARD9 Mutation.

Front. Immunol. 12:685546. doi: 10.3389/fimmu.2021.685546

\section{Case Report: Talaromyces marneffei Infection in a Chinese Child With a Complex Heterozygous CARD9 Mutation}

\author{
Hongjun Ba ${ }^{1,2}$, Huimin Peng ${ }^{1}$, Liangping Cheng ${ }^{1}$, Yuese $\mathrm{Lin}^{1}$, Xuandi $\mathrm{Li}^{1}$, Xiufang $\mathrm{He}^{1}$, \\ Shujuan $L^{1}{ }^{1}$, Huishen Wang ${ }^{1}$ and Youzhen Qin ${ }^{1,2 *}$ \\ ${ }^{1}$ Department of Paediatric Cardiology, Heart Centre, The First Affiliated Hospital, Sun Yat-sen University, Guangzhou, China, \\ 2 Key Laboratory on Assisted Circulation, Ministry of Health, Guangzhou, China
}

Talaromyces marneffei (TM) infection is rarely seen in clinical practice, and its pathogenesis may be related to deficiency in antifungal immune function. Human caspase recruitment domain-containing protein 9 (CARD9) is a key molecule in fungal immune surveillance. There have been no previous case reports of TM infection in individuals with CARD9 gene mutations. Herein, we report the case of a 7 -month-old Chinese boy who was admitted to our hospital with recurring cough and fever with a papular rash. A blood culture produced TM growth, which was confirmed by metagenomic next-generation sequencing. One of the patient's sisters had died of TM septicaemia at 9 months of age. Whole exome sequencing revealed that the patient had a complex heterozygous CARD9 gene mutation with a c. $1118 \mathrm{G}>$ C p.R373P variation in exon 8 and a c.610C > T p.R204C variation in exon 4. Based on the culture results, voriconazole antifungal therapy was administered. On the third day of antifungal administration, his temperature dropped to within normal range, the rash gradually subsided, and the enlargement of his lymph nodes, liver, and spleen improved. Two months after discharge, he returned to the hospital for a follow-up examination. His general condition was good, and no specific abnormalities were detected. Oral voriconazole treatment was continued. Unexplained TM infection in HIVnegative individuals warrants investigation for immune deficiencies.

Keywords: Talaromyces marneffei infection, CARD9 mutation, fungal immunodeficiency, CARD9 deficiency, immunodeficiency disease

\section{INTRODUCTION}

Talaromyces marneffei (TM) is an opportunistic pathogen. TM is a member of the family Trichocomaceae, order Eurotiales, class Eurotiomycetes, division Ascomycota. It is the only member of the genus Talaromyces and is considered an important human pathogen. TM infection is clinically rare and occurs mainly in individuals with HIV infection. In HIV-negative individuals, TM infection occurs mainly in patients with congenital immune deficiencies (1). Many genetic conditions are associated with congenital immune deficiencies, including CARD9 (caspase recruitment domain-containing protein 9) gene mutations. Human CARD9 is a key molecule in 
fungal immune surveillance (2). In the year 2013, the primary genetic aetiology of deep dermatomycosis was identified as homozygous loss of function mutations in CARD9 in 17 patients from eight unrelated North African families (3). One homozygous premature stop codon mutation (Q289*) was found in 15 patients from seven unrelated families in Algeria and Tunisia, and one homozygous missense mutation (R101C) was found in two Moroccan siblings (4). The CARD9Q289* mutation was also recently identified in an Egyptian patient with extensive skin and nail mycosis (5). Experiments in mice have shown that CARD9 is an adaptor molecule, expressed primarily in macrophages and myeloid dendritic cells, and plays a central role in antifungal defence by receiving signals from several C-type lectin-like receptors and stimulating proinflammatory responses (6). In addition, CARD9-deficient cells have been shown to exhibit selective impairment of tumour necrosis factor- $\alpha$ and interleukin- 6 production when stimulated by fungal antigens (6-8). Several cases of CARD9 mutations combined with fungal infections such as candidiasis have been reported, but there are no previous reports of CARD9 gene mutation combined with TM infection. In this report, we describe an infant with CARD9 mutation combined with TM infection in order to raise clinicians' awareness of CARD9 mutations as a possible cause of vulnerability to fungal infections.

\section{CASE DESCRIPTION}

A 7-month-old Chinese boy was admitted to our hospital with a 2month history of a recurring cough, fever for nine days, and a worsening rash for two days. The local hospital had administered a variety of antimicrobials prior to referral, but the patient's condition had not improved. Physical examination on admission revealed facial and bodily maculopapules, reddish-brown papules covering the limbs, swelling of the lower limbs, cervical lymphadenopathy, and faint wet rales on auscultation of both lungs. His heart rate was 160-170 bpm with normal heart sounds. Hepatosplenomegaly was present, and neurological examination was normal. He had previously been observed to have left axillary lymph node enlargement after BCG inoculation at birth. The patient was the third child in the family and had two older sisters. The second sister had died of recurrent fever with severe sepsis at 9 months of age, with TM produced in bone marrow and blood cultures.

Chest computed tomography (CT) examination revealed bilateral pulmonary infiltrates. Abdominal CT revealed significant hepatosplenomegaly and multiple enlarged abdominal lymph nodes. Colour Doppler ultrasound of the heart showed enlargement of the left ventricle and a small pericardial effusion, but no significant dilation of the left and right coronary arteries.

Laboratory tests revealed a serum C-reactive protein (CRP) level of $272 \mathrm{mg} / \mathrm{L}$ (reference range, $<10 \mathrm{mg} / \mathrm{L}$ ) and serum procalcitonin (PCT) level of $2.73 \mathrm{ng} / \mathrm{mL}$ (reference range, $<0.05 \mathrm{ng} / \mathrm{mL}$ ). Routine haematologic testing revealed the following: white blood cell count (WBC), $33 \times 10^{9}$ cells/L (reference range, $4-10 \times 10^{9}$ cells/L); neutrophil count, $16.13 \times 10^{9}$ cells/L; lymphocyte count, $12.85 \times 10^{9}$ cells $/ \mathrm{L}$; and monocyte count, $1.24 \times 10^{9}$ cells/L. The serum $\mathrm{N}$-terminal pro b-type natriuretic peptide level was $979.2 \mathrm{pg} / \mathrm{mL}$ (reference range, $0-84 \mathrm{pg} / \mathrm{mL}$ ). Serological tests for HIV were negative. Cellular immune function tests revealed CD3+, 82.9\%; CD3 +CD4+, 50.8\%; CD3+CD8+, 29.8\%; CD19+, 12.8\%; and natural killer cells, $4 \%$. Results of humoral immune function testing were within normal limits. Th1/Th2/Th17 cytokine detection showed the following: tumour necrosis factor level, $1.48 \mathrm{pg} / \mathrm{mL}$ (reference range, $0-4.6 \mathrm{pg} / \mathrm{mL}$ ); interleukin-6 level, $4.6 \mathrm{pg} / \mathrm{mL}$ (reference range, $0-5.3 \mathrm{pg} / \mathrm{mL}$ ); interferon-gamma level, $2.66 \mathrm{pg} / \mathrm{mL}$ (reference range, $0-7.42 \mathrm{pg} / \mathrm{mL}$ ); interleukin17 level, $6.49 \mathrm{pg} / \mathrm{mL}$ (reference range, 0-20.6 pg/mL); interleukin-10 level, $8.59 \mathrm{pg} / \mathrm{mL}$ (reference range, 0-4.91 pg/ $\mathrm{mL}$ ); and interleukin-2 level, $1.11 \mathrm{pg} / \mathrm{mL}$ (reference range, 0-5.71 $\mathrm{pg} / \mathrm{mL}$ ). A blood culture produced growth of TM. No growth was found on bone marrow microbial culture.

Metagenomic next-generation sequencing ( $\mathrm{mNGS)}$ of pathogenic blood microorganisms confirmed TM infection (Figure 1). Bone marrow cytology and culture showed no obvious abnormalities.

DNA samples from the patient's family members were collected with their consent (Figure 2). CARD9 was amplified using specific primers. The patient was found to have a complex heterozygous CARD9 genotype with a c.1118G $>$ C p.R373P variation in exon 8 and a c.610C $>$ T p.R204C variation in exon 4 (Figure 3). The Sanger method was used to verify the results. The patient's father and paternal grandmother both had the c.1118G >C p.R373P variation in exon 8, but neither fell ill. In addition, the patient's mother had a c.610C $>$ T p.R204C variation in exon 4 but had no medical history of note(Figure 3). Sorting Intolerant From Tolerant (SIFT) is a tool for predicting the harm of genetic variation. The SIFT score ranges from 0 to 1 , and a score $<0.05$ is predicted to be deleterious. The SIFT scores of variations c.1118G $>$ C p.R373P and c.610C $>$ T p.R204C were 0.007 and 0 , respectively. The SIFT prediction hints were both damaging. The CARD9 gene mutation with a c.1118G >C p.R373P variation in exon 8 has been reported previously, but the c.610C $>$ T p.R204C variation in exon 4 has not.

Other pathogens detected by mNGS were considered as background organisms due to low copy numbers. Because the local hospital had administered potent anti-bacterial treatment with no results, we considering the likelihood of bacterial infection to be low; therefore, at first, we administered only cefuroxime as an empirical treatment. We administered voriconazole antifungal therapy based on the TM culture susceptibility testing results. On the third day of administration, the patient's body temperature returned to normal, rash gradually subsided, lymphadenopathy and hepatosplenomegaly improved, and heart rate returned to normal. After seven days of voriconazole treatment, his WBC and serum levels of CRP, PCT, and NT-proBNP were all within normal limits.

Two months after discharge, the patient returned to the hospital for a follow-up examination. His general condition was normal without any specific abnormalities. The treatment with oral voriconazole was continued. 
A

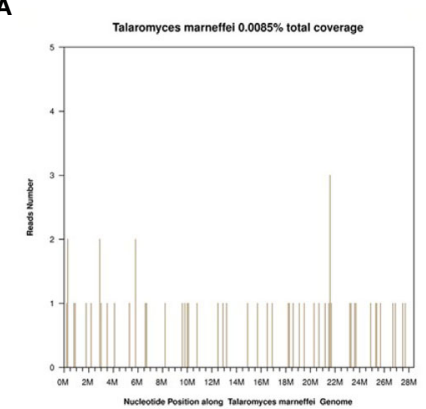

B

pathogenic sequences in serums $(\mathrm{N}=529$ reads)

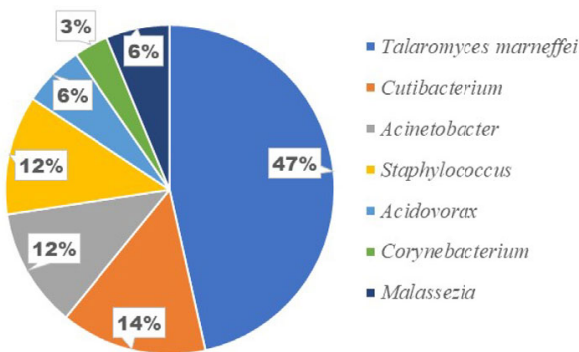

FIGURE 1 | Confirmation of talaromyces marneffei specific amplification from plasma by next-generation sequencing. (A) shows the reads mapped to talaromyces marneffei derived from NGS data. A total of 248 reads mapped to talaromyces marneffei in the reference database which contains about 8000 pathogen genomes, and got a total coverage of $0.0085 \%$ respectively. (B) shows the distribution of bacterial sequences ( $\mathrm{N}=529$ reads) identified in the patient's plasma included Talaromyces marneffei ( $\mathrm{N}=248 ; 47 \%)$, Cutibacterium, Acinetobacter, Staphylococcus, Acidovorax, Corynebacterium, Malassezia.

A

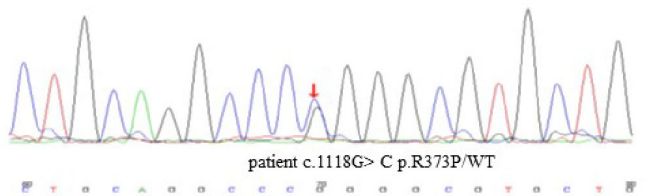

$\downarrow$
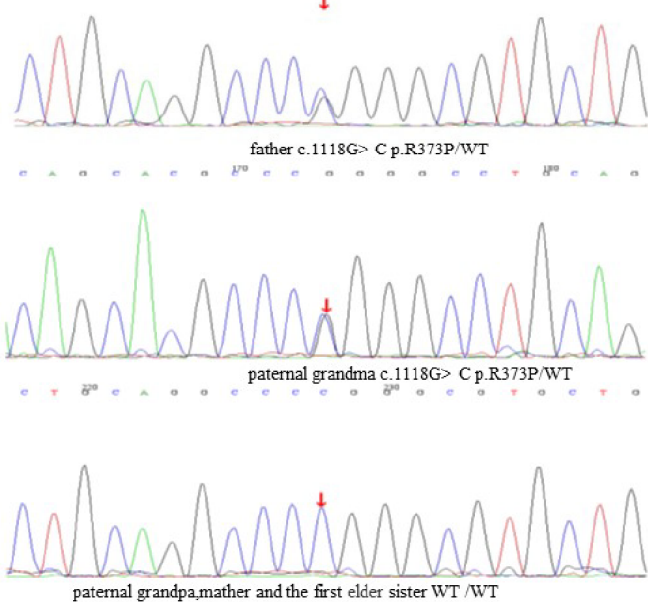

B
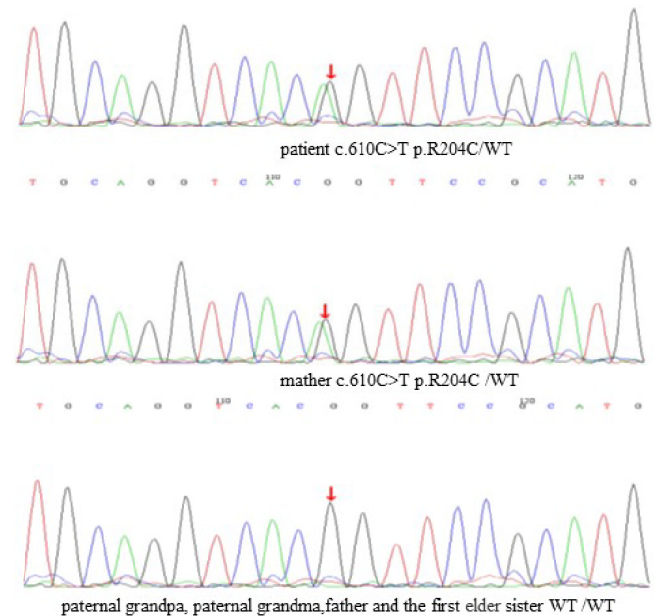

FIGURE 2 | Family evaluation of CARD9. (A) c.1118G>C p.R373P variation in exon 8. (B) c.610C>T p.R204C variation in exon 4.

\section{DISCUSSION}

TM infection is endemic in tropical regions, especially Thailand, Vietnam, northeastern India, Southern China, Hong Kong, Taiwan, Laos, Malaysia, Myanmar, Cambodia and Laos (9). TM is a deep pathogenic fungus first isolated by Capponi et al. in 1956 from the liver of the Vietnamese bamboo mouse (10). The bamboo mouse carries TM, and its excrement travels through water to contaminate soil. Humans can be infected through the skin, the respiratory tract, or the gastrointestinal tract. In the past, TM infection has been recognized as one of the three major opportunistic infections of
AIDS, along with tuberculosis and cryptococcosis (11). In recent years, improved treatment of HIV infection through aggressive antiretroviral therapy and other measures to control the HIV/AIDS epidemic have led to changes in the epidemiology of TM infection and an increasing number of non-HIV infected patients with other immunocompromised conditions. CARD9 mutation is one of the important causes of primary immunodeficiency disease (8).There have been no reports of TM infection patients with CARD9 mutations in children.

TM can invade multiple organs of the body, proliferate in macrophages, and spread through the endothelial network, 


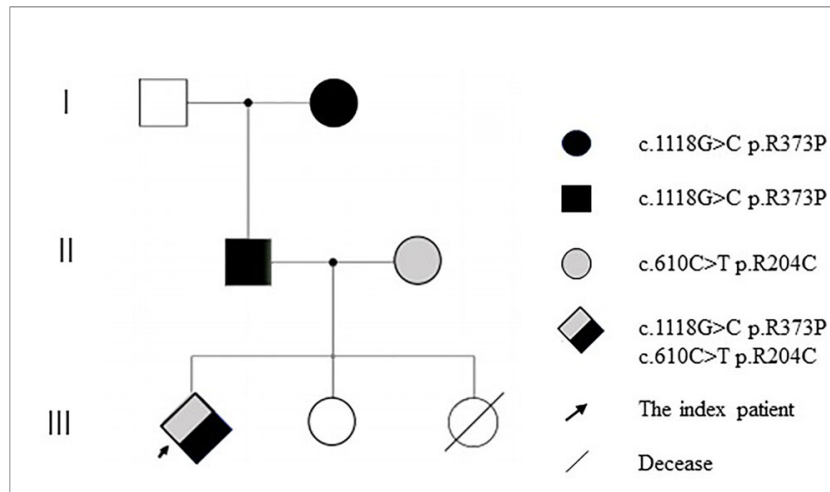

FIGURE 3 | Pedigree of CARD9 mutations (Due to death, no genetic analysis was performed for the second elder sister).

especially in the blood, bone marrow, skin, lungs, and reticuloendothelial tissue (12). Similar to other intracellular pathogens, T-lymphocyte-derived cytokines activate macrophages, especially those that respond to Th1, such as interleukin (IL)-12, IFN- $\gamma$, and tumor necrosis factor (TNF)- $\alpha$, which are important cytokines for host defense against TM infection (13). This is supported by the observation that in mice with reduced $\mathrm{T}$ lymphocytes, TM infection is always fatal, while in healthy mice the fungus can be cleared within three weeks $(14,15)$. There was no significant increase in TNF and IL in this patient, so the clinical presentation was very severe. The patient presents with high fever, rash, hepatosplenomegaly, and cervical lymphadenopathy. These were consistent with the possible mechanisms of TM infection reported in the above literature.

With general bacterial infectious diseases, serum CRP and PCT levels will be significantly increased, and in fungal infectious diseases, they are rarely increased. However, when fungal sepsis occurs, serum CRP and PCT levels will significantly increase. This suggests that in clinical practice, in addition to bacterial infection, the possibility of fungal sepsis should be considered for patients with significantly abnormal serum CRP and PCT levels. Due to significantly elevated CRP and PCT in our patient, the local hospital provided empirical anti-bacterial treatment with vancomycin and meropenem, but the effect was not ideal, and recurrent high fever persisted. Therefore, fungal sepsis was suspected. Further mNGS identified the pathogen as $\mathrm{TM}$, and the patient improved after antifungal treatment with voriconazole. Therefore, in children with fever of unknown origin and multi-system infection in regions with high incidence of TM infection, TM should be considered in the differential diagnosis when screening for fungal infection. TM is the only rare pathogen with temperature biphasic type in Penicillium. Routine cultures are prone to false negatives, and mNGS is a new technology for detection of pathogens using metagenomic next-generation sequencing. mNGS can be used for in-depth and rapid identification of pathogens without culture, which is more sensitive than traditional culture methods. Therefore, Pathogens should be identified as soon as possible using mNGS so that effective targeted treatment can be initiated.

TM infection is relatively rare clinically and mainly occurs in patients with acquired immunodeficiency. It has been reported that $30 \%$ of patients with AIDS are infected with TM (16). However, our patient repeatedly tested HIV-negative, and the history of an infant sister who had died due to TM infection suggested a possible genetic immunodeficiency in the patient's family. Therefore, we further conducted family medical whole exon gene sequencing, and the test indicated that the child had a compound heterozygous CARD9 gene mutation.

$C A R D$ 9, a member of the CARD family, is an important linking protein found by Bertin et al. (17). Since the first report of CARD9 mutation in patients with chronic cutaneous and mucosal candidiasis in 2009 (18), there have been successive reports indicating that CARD9 mutations can significantly increase the susceptibility to a variety of fungi. CARD 9 can effectively integrate the recognition signals of various natural immune receptors and plays an important role in antifungal immunity. As an important mediator of C-type lectin receptor and other receptor pathways, it activates inflammatory signalling pathways such as nuclear factor $\kappa \mathrm{B}$, and then activates downstream signalling molecules to promote the production of inflammatory cytokines (19). CARD9 mutations are autosomal recessive, and homozygous mutations are the most pathogenic; complex heterozygous mutations have also been reported. At present, it is known that patients with CARD9 gene mutation are prone to fungal infection, and the common pathogens are Candida, Aspergillus, and dermatophytes (20-22).There have been no reports of CARD9 mutation in patients with TM infection.

Medical whole exon sequencing showed a complex heterozygous CARD9 mutation, one of which was c.1118G $>C \mathrm{p} . \mathrm{R} 373 \mathrm{P}$, which has been reported in the literature. The findings demonstrate that through impaired phagocytic killing, human CARD9 deficiency results in a selective defect in the host defence against invasive infection (23). C.610C $>\mathrm{T}$ p.R204C was a new and previously unreported mutation site, and its pathogenicity was predicted using software. Further tracing of the family revealed that the mutation c.1118G $>$ C p.R373P was inherited from the patient's father, who in turn inherited it from the paternal grandmother. C.610C $>$ T p.R204C was inherited from the patient's mother. From a genetic perspective, we speculate that the patient's second sister may have also had a complex heterozygous CARD 9 mutation, dying as a result of TM infection. The cytokine analysis of the child showed no significant increase in IL- 6 and TNF, suggesting that the presence of a complex heterozygous CARD9 mutation may lead to decreased antifungal immunity and a susceptibility to TM infection.

In conclusion, clinicians should be alert to the possibility of primary immunodeficiency when TM infection occurs in HIVnegative individuals from endemic areas. mNGS and immunodeficiency related gene test should be completed as soon as possible for suspected patients.

\section{ETHICS STATEMENT}

Written informed consent was obtained from patients' parents for publication of this case report and any potentially identifying information. The work was exempt from the ethics committee review/approval. 


\section{AUTHOR CONTRIBUTIONS}

All authors listed have made a substantial, direct, and intellectual contribution to the work, and approved it for publication.

\section{REFERENCES}

1. Si Z, Qiao J. Talaromyces Marneffei Infection. N Engl J Med (2017) 377:2580. doi: 10.1056/NEJMicm1704164

2. Drummond RA, Franco LM, Lionakis MS. Human CARD9: A Critical Molecule of Fungal Immune Surveillance. Front Immunol (2018) 9:1836. doi: 10.3389/fimmu.2018.01836

3. Lanternier F, Cypowyj S, Picard C, Bustamante J, Lortholary O, Casanova JL, et al. Primary Immunodeficiencies Underlying Fungal Infections. Curr Opin Pediatr (2013) 25:736-47. doi: 10.1097/MOP.0000000000000031

4. Lanternier F, Pathan S, Vincent QB, Liu L, Cypowyj S, Prando C, et al. Deep Dermatophytosis and Inherited CARD9 Deficiency. N Engl J Med (2013) 369:1704-14. doi: 10.1056/NEJMoa1208487

5. Jachiet M, Lanternier F, Rybojad M, Bagot M, Ibrahim L, Casanova JL, et al. Posaconazole Treatment of Extensive Skin and Nail Dermatophytosis Due to Autosomal Recessive Deficiency of CARD9. JAMA Dermatol (2015) 151:1924. doi: 10.1001/jamadermatol.2014.2154

6. Hsu YM, Zhang Y, You Y, Wang D, Li H, Duramad O, et al. The Adaptor Protein CARD9 is Required for Innate Immune Responses to Intracellular Pathogens. Nat Immunol (2007) 8:198-205. doi: 10.1038/ni1426

7. Lanternier F, Barbati E, Meinzer U, Liu L, Pedergnana V, Migaud M, et al. Inherited CARD9 Deficiency in 2 Unrelated Patients With Invasive Exophiala Infection. J Infect Dis (2015) 211:1241-50. doi: 10.1093/infdis/jiu412

8. Lanternier F, Mahdaviani SA, Barbati E, Chaussade H, Koumar Y, Levy R, et al. Inherited CARD9 Deficiency in Otherwise Healthy Children and Adults With Candida Species-Induced Meningoencephalitis, Colitis, or Both. J Allergy Clin Immunol (2015) 135:1558-68 e2. doi: 10.1016/j.jaci.2014.12.1930

9. Vanittanakom N, Cooper CRJr., Fisher MC, Sirisanthana T. Penicillium Marneffei Infection and Recent Advances in the Epidemiology and Molecular Biology Aspects. Clin Microbiol Rev (2006) 19:95-110. doi: 10.1128/CMR.19.1.95-110.2006

10. Capponi M, Segretain G, Sureau P. Penicillosis From Rhizomys Sinensis. Bull Soc Pathol Exot Filiales (1956) 49:418-21.

11. Hu Y, Zhang J, Li X, Yang Y, Zhang Y, Ma J, et al. Penicillium Marneffei Infection: An Emerging Disease in Mainland China. Mycopathologia (2013) 175:57-67. doi: 10.1007/s11046-012-9577-0

12. Guo J, Li BK, Li TM, Wei FL, Fu YJ, Zheng YQ, et al. Characteristics and Prognosis of Talaromyces Marneffei Infection in Non-HIV-Infected Children in Southern China. Mycopathologia (2019) 184:735-45. doi: 10.1007/s11046019-00373-4

13. Sisto F, Miluzio A, Leopardi O, Mirra M, Boelaert JR, Taramelli D. Differential Cytokine Pattern in the Spleens and Livers of BALB/c Mice Infected With Penicillium Marneffei: Protective Role of Gamma Interferon. Infect Immun (2003) 71:465-73. doi: 10.1128/IAI.71.1.465-473.2003

\section{FUNDING}

The study was supported by Guangdong Basic and Applied Basic Research Foundation (2020A1515010184).

14. Kudeken N, Kawakami K, Kusano N, Saito A. Cell-Mediated Immunity in Host Resistance Against Infection Caused by Penicillium Marneffei. J Med Vet Mycol (1996) 34:371-8. doi: 10.1080/02681219680000671

15. Kudeken N, Kawakami K, Saito A. CD4+ T Cell-Mediated Fatal Hyperinflammatory Reactions in Mice Infected With Penicillium Marneffei. Clin Exp Immunol (1997) 107:468-73. doi: 10.1046/j.1365-2249.1997.d01-945.x

16. Limper AH, Adenis A, Le T, Harrison TS. Fungal Infections in HIV/AIDS. Lancet Infect Dis (2017) 17:e334-43. doi: 10.1016/S1473-3099(17)30303-1

17. Bertin J, Guo Y, Wang L, Srinivasula SM, Jacobson MD, Poyet JL, et al. CARD9 Is a Novel Caspase Recruitment Domain-Containing Protein That Interacts With BCL10/CLAP and Activates NF-kappa B. J Biol Chem (2000) 275:41082-6. doi: 10.1074/jbc.C000726200

18. Glocker EO, Hennigs A, Nabavi M, Schaffer AA, Woellner C, Salzer U, et al. A Homozygous CARD9 Mutation in a Family With Susceptibility to Fungal Infections. N Engl J Med (2009) 361:1727-35. doi: 10.1056/NEJMoa0810719

19. Drummond RA, Saijo S, Iwakura Y, Brown GD. The Role of Syk/CARD9 Coupled C-Type Lectins in Antifungal Immunity. Eur J Immunol (2011) 41:276-81. doi: 10.1002/eji.201041252

20. Du B, Shen N, Hu J, Tao Y, Mo X, Cao Q. Complete Clinical Remission of Invasive Candida Infection With CARD9 Deficiency After G-CSF Treatment. Comp Immunol Microbiol Infect Dis (2020) 70:101417. doi: 10.1016/j.cimid.2020.101417

21. Zhang Y, Huang C, Song Y, Ma Y, Wan Z, Zhu X, et al. Primary Cutaneous Aspergillosis in a Patient With CARD9 Deficiency and Aspergillus Susceptibility of Card9 Knockout Mice. J Clin Immunol (2021) 41:427-40. doi: 10.1007/s10875-020-00909-0

22. Nazarian RM, Lilly E, Gavino C, Hamilos DL, Felsenstein D, Vinh DC, et al. Novel CARD9 Mutation in a Patient With Chronic Invasive Dermatophyte Infection (Tinea Profunda). J Cutan Pathol (2020) 47:166-70. doi: 10.1111/cup.13574

23. Drewniak A, Gazendam RP, Tool AT, van Houdt M, Jansen MH, van Hamme JL, et al. Invasive Fungal Infection and Impaired Neutrophil Killing in Human CARD9 Deficiency. Blood (2013) 121:2385-92. doi: 10.1182/blood-2012-08450551

Conflict of Interest: The authors declare that the research was conducted in the absence of any commercial or financial relationships that could be construed as a potential conflict of interest.

Copyright (C) $2021 \mathrm{Ba}$, Peng, Cheng, Lin, Li, He, Li, Wang and Qin. This is an openaccess article distributed under the terms of the Creative Commons Attribution License (CC BY). The use, distribution or reproduction in other forums is permitted, provided the original author(s) and the copyright owner(s) are credited and that the original publication in this journal is cited, in accordance with accepted academic practice. No use, distribution or reproduction is permitted which does not comply with these terms. 